\title{
La integración del control y la evaluación de resultados en el proceso presupuestario: limitaciones y condiciones para su desarrollo
}

\section{Eduardo Zapico-Goñi *}

\section{Introducción}

Existe un sentimiento común de desorientación por los débiles resultados y la dificultad de avanzar en la introducción de las reformas tradicionales de control y gestión del gasto, sobre todo en lo que se refiere a la idea de reasignar los recursos disponibles para ejercicios futuros en función de los resultados conseguidos en la gestión del ejercicio precedente. Desde hace más de dos décadas se viene recogiendo información sobre los intentos de implantar sistemas o modelos racionales de presupuesto (Presupuesto por Programas, Presupuesto en Base Cero, entre otros) o reformas similares. Los resultados de una amplia encuesta del Instituto Internacional de Ciencias Administrativas mostraron que «... en el curso de las últimas décadas no ha habido jamás una aplicación sistemática de los elementos de un sistema presupuestario integrado, a excepción del caso del Ministerio de Defensa en los Estados Unidos» (ParISIS, 1980). En términos generales los intentos de reforma fracasaron pero en relación a las grandes expectativas creadas y a la manera mecanicista de entender la racionalidad en el presupuesto. Algunos de los esfuerzos realizados no han sido totalmente infructuosos, especialmente en las iniciativas de reforma presupuestaria en los últimos años orientadas de manera más pragmática para la aplicación parcial de algunos de los elementos de las técnicas racionales de presupuestación. Parece haberse «llenado la copa» de las ideas e iniciativas de reforma presupuestaria (A. SCHCK, 1990), y se sigue trabajando en una línea de reforma muy parecida, intentando introducir lo que ahora se denomina un presupuesto por resultados (OCDE, 1996; J. BLONDAL, 2000).

El fracaso de las grandes reformas presupuestarias, la prolongada crisis económica en los años setenta y la expansión del liberalismo económico, entre otros factores, indujeron a muchos gobiernos a recortar directamente el gasto público a nivel agregado según sus prioridades o la viabilidad de cada recorte. Las medidas de disciplina fiscal propuestas en países de la OCDE, desde principios de los años noventa, han sido normas concretas para frenar el aumento del gasto público: límite agregado del gasto en relación con el PIB, límite por partidas funcionales, obligatoriedad de acompañar con contrapartidas las propuestas de modificaciones presupuestarias, no compensación de la inflación, endurecimiento de las condiciones de acceso al subsidio de desempleo, pensiones y otros derechos adquiridos (WLDAVSKY, 1993). Estas propuestas se han complementado con otras medidas (p. e., proyección a largo de escenarios presupuestarios, mayor rigidez en la ejecución del gasto a nivel agregado), en conjunto encaminadas a reforzar el papel de dominio del Ministerio de Finanzas frente a otros actores presupuestarios en todas las fases del ciclo presupuestario. 
En la Comunidad Europea también se ha establecido desde mediados de los años noventa un marco de disciplina presupuestaria que ayudará a cumplir las condiciones de convergencia requeridas por el Tratado de Maastricht para la Unión Económica y Monetaria. Los Estados miembros presentaron programas de convergencia con propuestas de reducciones drásticas en el gasto público. En la actualidad se está reforzando la disciplina fiscal mediante los Pactos de Estabilidad y Empleo y con normas más concretas en cada país (p. e., Proyecto de Ley de Estabilidad Presupuestaria, 2000) para frenar las propuestas de gasto de los departamentos gestores, controlar la disciplina de gasto de los distintos niveles de gobierno y consolidar la posición de dominio del Ministerio de Finanzas en todas las fases del ciclo presupuestario.

Estas medidas son necesarias para controlar el gasto, pero a medio y largo plazo su eficacia está muy condicionada por la propia inestabilidad e incertidumbre del contexto en que se aplican. Los ajustes a nivel macro presupuestario parecen estar teniendo un cierto éxito, pero está pendiente de corroborarse si las innovaciones en las instituciones y en el control y gestión presupuestario permitirán conseguir la sostenibilidad de la disciplina fiscal y se evitará el crecimiento descontrolado del gasto público cuando el ciclo económico sea menos favorable. La cuestión no está sólo en el dominio o la firmeza del Ministerio de Finanzas para recortar las cifras de gasto cada vez que negocia con los departamentos gestores los créditos presupuestarios para el ejercicio siguiente, además se necesita desarrollar la capacidad de controlar la realidad social y económica que presiona al alza el gasto público. Los sistemas de control, evaluación y gestión estratégica del gasto en este Ministerio y en toda la Administración son fundamentales para adaptar el comportamiento presupuestario de manera coherente a los cambios del entorno (ZAPICO, 2001).

Es cierto que se necesita reforzar la posición de las instituciones centrales de presupuesto. Pero este refuerzo no debe estar sólo dirigido a potenciar el papel del Ministerio de Finanzas en lo que se refiere a la imposición de normas fiscales, a la resolución de conflictos en las negociaciones presupuestarias y a la inspección tradicional del gasto, sino más bien a proporcionar un liderazgo estratégico en la gestión del gasto más allá de la mera proyección macro-econométrica (elaboración de escenarios presupuestarios y Pactos de Estabilidad) y la aprobación de exigentes normas presupuestarias. La cara oculta del presupuesto como instrumento de gestión y como marco para la evaluación del gasto público es una asignatura pendiente de la reforma presupuestaria que es necesaria para conseguir una disciplina presupuestaria sostenida. A medio y largo plazo la evaluación de políticas públicas puede tener consecuencias importantes en el funcionamiento de la Administración. Unos sistemas de control y evaluación orientados a la adaptación estratégica del presupuesto pueden ser de gran utilidad para el Ministerio de Finanzas.
La evaluación de programas no proporciona una solución inmediata contra la indisciplina presupuestaria a nivel macro. Pero puede orientar al Departamento de Finanzas en el diseño de un marco de referencia más legítimo para imponer con eficacia una mayor disciplina de gasto (fijación de techos de gasto más realista, distribución de límites agregados entre prioridades sectoriales, etc.). Además, el seguimiento de resultados y la evaluación, a nivel de centro gestor, puede ser de gran utilidad para que este se adapte a una situación más restrictiva, en la que se necesita ser más eficiente y reasignar recursos dentro del límite presupuestario impuesto desde el Ministerio de Finanzas.

En principio nadie se opone a la idea de utilizar la evaluación de programas o políticas públicas a la hora de redistribuir los recursos disponibles. Han sido múltiples los estudios realizados desde los años sesenta en favor de introducir la técnica de presupuesto por programas o los sistemas integrados de presupuestación (D. Novick, 1969; A. SHICK, 1978, etc.). El objetivo de las reformas presupuestarias basadas en estos modelos no era otro sino el de reasignar recursos en base al análisis de los resultados de la gestión de los programas presupuestarios. Aunque estos modelos de reforma se han abandonado, en muchos países se siguen proponiendo modelos muy parecidos con términos casi sinónimos: i. e. el presupuesto por resultados o la integración de la medición y evaluación de resultados en la reasignación de recursos (PUmA, 1997, p. 36).

Pero la evaluación y el presupuesto por resultados representa también un desafío importante. El presupuesto se puede percibir metafóricamente como una moneda con una cara en la que se refleja su aspecto técnico y racional y con una cruz en la que se refleja su aspecto negociado. La evaluación de programas no deja de ser un riesgo para el centro de presupuestos, pues permite al gestor argumentar a favor de la necesidad de un aumento del gasto.

En este artículo se plantea y discute la importancia y posibilidad de la integración de las funciones de control, la evaluación y el presupuestario. ¿Qué ocurre de hecho en la realidad? ¿Hay alguna experiencia conocida en la que este ejercicio de «racionalidad presupuestaria» se realice? ¿Hasta qué punto es posible la presupuestación por resultados? ¿En qué medida es apropiado el modelo presupuestario propuesto como referencia para la reforma? En las próximas secciones se analizan algunas líneas de respuesta a estas preguntas con el fin de replantear la discusión sobre las posibilidades de utilizar el producto del control por resultados y la evaluación de programas en el proceso presupuestario. Al final del artículo se analizan las condiciones necesarias para conseguir esta integración, sugiriendo las iniciativas que se consideran más relevantes para proporcionar una mayor coherencia de estas funciones y el proceso presupuestario. 


\section{El control y la evaluación de resultados en la práctica presupuestaria}

En la práctica, el enlace de la evaluación de programas y el proceso presupuestario ha resultado ser más difícil de aplicar de lo que se esperaba. Las evidencias empíricas recogidas en diversos estudios para distintos niveles de gobierno así lo corroboran. En una investigación realizada sobre las experiencias prácticas de varios países desarrollados (Estados Unidos, Reino Unido, Canadá, Alemania, España, Suecia y Finlandia) indican que incluso en Suecia, donde se ha reconocido la necesidad y se han hecho importantes esfuerzos de integrar de manera regular y sistemática la evaluación de resultados en la elaboración del presupuesto, su aplicación ha sido débil e infrecuente (Gray, JenKIns y SegsworTH, 1993). Otros estudios realizados en países Nórdicos han coincidido con estos resultados (P. OvRelid, 1997). Estudios más recientes se manifiestan en la misma línea. Las dificultades de la implantación del presupuesto por resultados y otras reformas de la gestión pública han sido subestimadas (T. VerHeIEn y D. COOMBES, 1998).

Gray, JenKInS y SEGSWORTH analizan la integración de estas funciones desde cuatro puntos de vista complementarios, según el tipo de integración. En primer lugar valoran el grado de integración desde una perspectiva funcional. La integración funcional puede medirse por el grado de utilidad o consistencia del resultado de una actividad para con la otra y viceversa. El presupuesto y la evaluación son funciones muy distintas con productos diferentes que en realidad reflejan diferentes racionalidades legítimas.

La mayoría de los casos analizados en este estudio no presentan evidencias de que exista coherencia funcional entre el presupuesto y la evaluación de políticas públicas. La asignación de recursos presupuestarios está movida por una convicción doctrinal (ideología de mercado) más que por un esfuerzo de concepción o evaluación de las políticas públicas. En el estudio se demuestra que el valor añadido por cada una de estas funciones a las otras es muy débil. La cuestión no es simple y meramente técnica. Su integración requiere un esfuerzo continuo de coordinación que aceptando la pluralidad de perspectivas e intereses de cada grupo tienda a aproximarlas.

La falta de integración se analiza en este estudio también desde otros puntos de vista. Por ejemplo, la integración organizacional o grado de cohesión de las estructuras y procesos en los que se llevan a cabo el presupuesto y la evaluación que lógicamente también influye. La claridad y coherencia en el diseño de roles y responsabilidades de los actores que componen la red de organizaciones que participan en el ciclo presupuestario, entendido de manera amplia, es un aspecto fundamental en este sentido.

Pues bien, el estudio dirigido por GraY y otros vuelve a mostrar que la distribución de competencias e interrelaciones entre los participantes en el proceso presupuestario no está bien definida en ninguno de los países analizados. No obstante, se reconoce que el presupuesto y la auditoría están relativamente mejor integrados en algunos países (Alemania, España, Francia).

Otro tipo de integración considerado es la integración informativa o grado en el que se comparten sistemas de información y comunicación, de acceso común o con bases de datos coherentes, compatibles, etc. De nuevo la integración es muy limitada o inexistente. Aunque en los últimos años se han realizado avances considerables respecto a los sistemas de medición de resultados e información para la toma de decisiones, no hay evidencias claras de que existan marcos o canales comunes que faciliten la comunicación entre la evaluación y el presupuesto. En el estudio se observa que en Suecia los sistemas de información que se utilizan en la evaluación de las políticas públicas se usan también para el proceso presupuestario.

Por último, y quizás una de las más importantes, está la perspectiva socio-organizacional. La integración social puede entenderse como la homogeneidad en la cultura, preparación, experiencia y conocimiento del personal responsable de realizar estas funciones. Los resultados muestran que existe una preparación similar en el personal de presupuesto y el control en Alemania y en España, dada la tradición legal y la formación similar que reciben. Pero la evaluación queda fuera de estas consideraciones. La integración de la evaluación y el presupuesto exige el desarrollo de una cultura profesional y administrativa común o al menos semejante de manera que se abra espacio suficiente para la cooperación entre las distintas comunidades de expertos. En ningún país se dan las condiciones adecuadas para garantizar una comunicación eficaz entre ambas comunidades según el estudio mencionado. Sin embargo, en Suecia se han realizado programas de formación conjunta entre evaluadores y responsables del presupuesto. En este país se ha dado la aparente paradoja de que la propia institucionalización de la evaluación parece haber establecido barreras para su apertura a otras funciones tradicionalmente más arraigadas en la Administración como el presupuesto.

A nivel de gobiernos regionales o subcentrales las investigaciones realizadas ofrecen resultados parecidos. Las conclusiones de un estudio sistemático realizado por la General Accounting Office (Oficina de Auditoría del Congreso) y la Congressional Budget Office (Oficina de Presupuesto del Congreso) en los Estados Unidos, sobre el proceso presupuestario 
en los cinco Estados que habían realizado un mayor esfuerzo y que se consideraban más avanzados en la introducción de técnicas presupuestarias, mostraron que las Administraciones implicadas ofrecían pocas evidencias de que los resultados de la gestión influyeran en la asignación de los recursos públicos durante el proceso presupuestario. En general se reconoce que, en esencia, el presupuesto en estos Estados se sigue realizando de manera incrementalista $(\mathrm{GAO}, 1993)$.

Las conclusiones no son tan negativas en la Administración local según los datos disponibles. En las últimas décadas ha habido una evolución clara y generalizada en cuanto a la utilización de las técnicas modernas del presupuesto público en los Estados Unidos. La utilización de técnicas como el presupuesto por programas, el control de eficiencia, etc., se consolida en la Administración local durante los años ochenta. A finales de los ochenta se aprecia una ligera caída en su utilización en la Administración local (M. OrTigueIRA, 1989; S. Botner, 1991; D. Cothran, 1993), pero a diferencia de lo ocurrido en la Administración central estas técnicas racionales de presupuestación no desaparecen del ámbito de lo local, sino que se consolidan o se mantienen. En general, las conclusiones del estudio de BOTNER, realizado sobre las prácticas presupuestarias de las ciudades de tamaño medio en los Estados Unidos, muestran que se mantiene un nivel de profesionalismo presupuestario elevado. Un ejemplo más reciente del avance en el uso de las técnicas racionales de presupuestación en la Administración local en los Estados Unidos es el caso de la ciudad de Sunnyvale (California) que utiliza un sistema comprehensivo de presupuesto por tareas y gestión por resultados (OECD, 1994, n. 5). En la Administración local europea la tendencia de la reforma presupuestaria y control de gasto ha sido similar en términos generales a lo mencionado para Estados Unidos (OECD, 1994, n. 3). En algunos países se han realizado experiencias extensas de presupuesto por resultados en la Administración local. Una buena ilustración es, por ejemplo, el caso de Holanda donde se declara su utilización en 200 ayuntamientos (OECD, 1997).

Pero incluso volviendo al nivel de gobierno central, los resultados observados en las investigaciones mencionadas no significan que los esfuerzos realizados en las Administraciones más avanzadas no hayan tenido ninguna recompensa. En el proceso presupuestario de algunos países (RU, Suecia, Países Bajos) se dispone ahora de más información y mucho más relevante que hace unos pocos años sobre niveles de economía y eficiencia en la gestión. En estos países el Tesoro o el Ministerio de Finan. zas, respectivamente, exigen a los centros gestores la presentación de indicadores de resultados al solicitar nuevos créditos de un año para otro. El nivel de estos indicadores se va gradualmente desarrollando. Se están haciendo esfuerzos para desarrollar una mayor conexión entre la eficiencia operativa y la asignación de recursos (OCDE, 1993). No obstante, la rela- ción entre el seguimiento de la gestión y la asignación de recursos está pendiente de realizarse. La información recientemente generada por organismos internacionales apunta también en la misma dirección al reconocer la dificultad que entraña el salto de una gestión presupuestaria por resultados operativos (por actividades y outputs) a otra por resultados en términos de efectos finales de las políticas públicas (outcomes or impacts) (BLON. DAL, 2000).

A pesar de estos débiles resultados hasta ahora conseguidos, la evaluación se sigue percibiendo hoy como de gran utilidad para promover una nueva cultura de gestión presupuestaria centrada en el resultado e impacto de los programas que en la formalidad de los procedimientos y en el ahorro inmediato. De los comentarios del comisario encargado de Control Financiero de la Comisión Europea se deduce que la evaluación se percibe como un buen instrumento para facilitar el tránsito de una cultura de gasto, donde gobernar o gestionar bien se confunde con aumentar los créditos presupuestarios, a una cultura de resultados, donde el directivo público se centra y es responsable por el grado de eficiencia y eficacia alcanzado al ejecutar su presupuesto (A. GradiN, 1996, p. 27).

Las reformas recientes insisten en el modelo de presupuestación por resultados. Sin embargo, algunos de sus componentes han sido introducidos ahora de una manera muy distinta y con un fin diferente. Los principales componentes de reforma son:

1) la definición de objetivos de gestión (estratégicos y operativos) y medición de resultados cuando sea posible y dirigido a cambiar la cultura tradicionalmente formalista de los participantes en la ejecución del presupuesto más que a la racionalización mecánica del proceso de asignación de recursos. El diseño y la utilización de los indicadores se realiza por los propios gestores y para su propio uso en el marco del presupuesto por resultados (OECD, 1995). Se pretende motivar al gestor para que cambie su estilo de decisión y comportamiento de gasto con mayor consciencia de coste y una visión clara sobre su productividad;

2) la descentralización en la gestión y mayor flexibilización de controles tradicionales del gasto. La intención final es proporcionar a los directivos públicos una mayor autonomía para gestionar por resultados sus programas presupuestarios (N. FLYNn y F. STREHL, 1996). La descentralización se percibe también como un paso para introducir y fomentar un estilo de decisión y un comportamiento del gestor público más parecido al de empresa privada (competitividad, consciencia de coste, eficiencia, etc.);

3) modificación de las relaciones entre las unidades centrales de presupuestos y los departamentos gestores mediante el uso de «contratos» presupuestarios (acuerdos presupuestarios) y adaptando los sistemas de responsabilidad por los resultados de la gestión su relación con la asignaciones de recursos, 
tanto a nivel central, local e intergubernamental (OECD, 1999) (Swedish Institute, 1996, citado en F. LoNGo, 1998).

Si bien se insiste en el esfuerzo y hay consenso sobre los beneficios que puede aportar el presupuesto por resultados y la evaluación de programas, hemos visto antes que hasta ahora han sido escasas las experiencias conocidas en el mundo en las que se haya ejercido la «racionalidad presupuestaria» de manera sistemática. ¿Por qué ocurre esto? ¿En qué medida y contexto es apropiado el modelo presupuestario propuesto como referencia para la reforma? ¿Cómo se podría avanzar con más solidez para integrar las funciones de presupuesto, control y evaluación?

\section{Análisis crítico del modelo de reforma: limitaciones y posibilidades de un sistema integrado de gestión presupuestaria}

\section{III.1. La naturaleza negociada y conflictiva como limitación a la racionalidad presupuestaria}

Se suelen mencionar razones como la falta de interés y liderazgo político, la no voluntad de ser medido y evaluado por parte de los gestores, la falta de colaboración de los profesionales de cada sector o política, la escasez de recursos, etc., para explicar la dificultad de implantar con éxito la reforma del presupuesto. Muchas de estas razones tienen su peso, pero existen otras de gran importancia, como la naturaleza negociada y conflictiva del proceso presupuestario, a las que tradicionalmente no se les ha prestado suficiente atención en el diseño de la reforma presupuestaria.

Es ingenuo creer que el control por resultados y la evaluación pueda sustituir la negociación política. El proceso de elaboración del presupuesto a alto nivel es un proceso de negociación en el que se enfrentan intereses contrapuestos. El presupuesto es negociación y conflicto (A. WLDAVSKY, 1979, p. 4) y seguirá siéndolo. La confrontación de intereses no es el reflejo de un mal funcionamiento del proceso presupuestario, sino una característica de la realidad en la que se desarrolla el proceso de elaboración del presupuesto: los recursos son escasos en rela- ción a las necesidades humanas, por naturaleza siempre insatisfechas.

También a nivel técnico el proceso presupuestario refleja las luchas de poder internas dentro de la Administración. La tradicional desconfianza entre el Departamento de Presupuestos y el gestor es otra de las razones que pueden explicar la escasa influencia de las técnicas de medición de resultados y evaluación en el proceso presupuestario. Al gestor le resulta sospechoso escuchar las reformas presupuestarias que se han propuesto desde organismos internacionales basadas con la idea de reasignar recursos desde actividades menos eficientes a las más eficientes (P. OvreLID, 1997, p. 7).

Aunque la retórica es atractiva, en la práctica es tradicional que el Departamento de Presupuestos no premie sino que de hecho penalice al gestor que por eficiencia o por otra causa consigue que le sobren unos recursos al final del ejercicio (Erkki LIKANEN, 1996, p. 35). En principio el gestor desconfía que siendo eficiente vaya a recibir más recursos en época de restricción presupuestaria.

El seguimiento de la gestión y la evaluación de los programas públicos no pueden entenderse ni pueden aplicarse de manera mecánica en el proceso presupuestario. $\mathrm{Ni}$ a nivel político ni a nivel técnico. Es necesario modificar los sistemas de incentivos que realmente motivan al gestor público. La reforma de los formatos y técnicas presupuestarias y la introducción de la evaluación de programas va detrás o a continuación de los cambios en el comportamiento o estilo de decisión en la asignación y utilización de los recursos y no al revés.

Pero esto no debe de entenderse como una conclusión derrotista como tradicionalmente se ha percibido desde la Administración o desde el mundo de la economía (STOCKMAN, 1984), sino como un desafío contextual con el que tienen que enfrentarse el Gobierno y la Administración al asignar y gestionar el gasto. El problema no está en la existencia del conflicto presupuestario, sino en la manera de confrontarlo. En algunas Administraciones hay una tendencia a evitar el conflicto. Éste se oculta y se le deja diluir en el tiempo, se decide no tomar ninguna decisión de reasignación de recursos por entenderse que no habría capacidad para gestionar una posible cadena de conflictos y agravios comparativos. Pero el conflicto puede confrontarse, se puede aprovechar la oportunidad que ofrece la crisis para aclarar y, si es posible, mejorar la situación para todos. El presupuesto es una oportunidad estratégica y recurrente para ejercitar la creatividad y desarrollar la capacidad de innovación en la gestión (ZAPICO, 1993).

Gran parte del camino para la reforma del presupuesto se habrá recorrido cuando los responsables de diseñarla y llevarla a cabo acepten que el estilo de decisión de políticas públicas, o el comportamiento y valores de decisión de los participantes en el proceso de decisión son tan importantes o más que las 
propias técnicas de control y presupuestación. Esto no es una conclusión pesimista sobre la utilidad del control y evaluación de programas. Estas dos funciones se pueden orientar en positivo y considerar como objetivo propio el fomentar una cultura de gasto o un estilo de decisión presupuestaria, incluidos los distintos tipos de solución de conflicto, adecuada al contexto presupuestario (METCALFE, 90; ZAPICO, 2001).

\section{III.2. Incoherencia entre modelo de gestión empresarial y la gestión presupuestaria en el sector público}

El sistema de gestión integrada del presupuesto se basa en el modelo tradicional de la gestión empresarial. La idea de aplicar los resultados del control y la evaluación de programas en la asignación de recursos va unida a la teoría racional de decisión. A mayor productividad o eficiencia de una actividad, mayores recursos se le deberían asignar en el ejercicio siguiente. El modelo se orienta a la maximización de beneficios o utilidad esperada y se basa en definitiva en el principio de competencia entre los actores presupuestarios que se supone estarían más motivados para mejorar los resultados de la gestión. Pero las fórmulas de «más productividad, más recursos» y «más con menos» (OECD, 1995 y 1997), aunque simples y atractivas, están siendo cada vez más cuestionadas por lo limitado, y en ocasiones no deseado, de sus efectos en la gestión pública.

El presupuesto por resultados, los contratos presupuestarios y las técnicas similares de presupuestación tienen una cierta utilidad el sector público. De hecho, se está utilizando en algunos países (Reino Unido, Países Bajos, NZ, etc.), en algunos servicios públicos (salud, policía) o incluso en los servicios centrales de la Administración de manera limitada, i.e. gastos de funcionamiento. Los valores competitivos tienen también importancia en la motivación de comportamientos productivos tanto en la empresa privada como en la Administración. Pero su utilidad está condicionada.

Desde un punto de vista de viabilidad organizativa, para que el modelo competitivo dé su fruto tiene que concederse una verdadera autonomía de acción al gestor público y disponerse de una capacidad de seguimiento y evaluación de resultados de la gestión. El departamento de presupuestos sólo puede conceder mayor autonomía de gasto en la medida que las unidades que reciben más poder y responsabilidad de gasto tengan capacidad de informar y demostrar los buenos resultados de su gestión financiera (N. FLynn y F. STREHLED, 1996). Hasta ahora, el impacto de la llamada Nueva Gestión Pública al intentar aumentar la eficiencia en la gestión del gasto público ha sido relativamente débil (T. VerHEIEN y D. COOMBES, 1998).

Desde un punto de vista conceptual, cada vez está más reconocida la necesidad de tener que analizar el contexto y adaptar el modelo de presupuesto a las características del mismo
(MetCalFe, 1990). Sin embargo, es un handicap en este sentido la poca capacidad que existe en la Administración para aprender y adaptar las iniciativas de reforma llevadas a cabo (OLSEN y Peters, 1996). Hasta mediados de los noventa ningún país había evaluado en profundidad el impacto en la Administración consecuencia de las recientes reformas presupuestarias. Hasta ahora estas evaluaciones han sido ad boc e inadecuadas en la mayoría de los países debido principalmente a problemas de medición y la sensibilidad política del tema (OCDE, 1993). «Los programas de reforma de la gestión pública normalmente no disponen de procedimientos de retroalimentación, evaluación y corrección o, si disponen de ellos, suelen ser despreciados y no se utilizan» (VERHEIEN y COOMBES, 1998).

Sin embargo, ciertos países han dado algunos pasos en esta línea. Australia realizó una evaluación comprehensiva de sus iniciativas de reforma en el sector público (Australia, Task Force on Management Improvement 1992). En 1994, el Cabinet Office realizó una investigación del impacto de las reformas realizadas en el Reino Unido (Sylvie Trosa, 1997) se basa en el Reino Unido. En Canadá, la Oficina del Controlador General (Tesoro) y el Auditor General (Parlamento) han evaluado el resultado de reformas llevadas a cabo para el desarrollo de la evaluación de programas (MAYNE y ZAPICO, ed. 1997).

Una de las condiciones necesarias en un contexto presupuestario para que tengan éxito la reforma clásica del sistema integrado de presupuestación es que haya «una cierta estabilidad de recursos» (OCDE, 1993, p. 5). Este tipo de dificultades ya se había anticipado en investigaciones académicas (Hofstede, 1981; MetCalfe, 1990). Aunque el modelo tradicional de reforma presupuestaria es útil en un contexto de estabilidad y certidumbre, puede ser inapropiado o insuficiente para el tipo de trabajo que se realiza en muchas organizaciones públicas, especialmente en los departamentos centrales y en las organizaciones de profesionales y en general en la red de organizaciones que participa en el proceso presupuestario, bajo gran complejidad y en una realidad «multifacética» (OLSEN y PETERS, 1996).

Las reformas centradas en la eficiencia y en la competición por los recursos han tenido un resultado limitado a la consecución de recorte de los gastos corrientes o de funcionamiento, relativamente reducidos. Dado el contexto de incertidumbre e inestabilidad, gran parte de los recursos públicos se están gestionando de manera eficiente pero están resolviendo problemas que han dejado de existir o se están proporcionando servicios que la sociedad no valora de la misma manera que años atrás. Esto es más fácil que ocurra en aquellas reformas presupuestarias enfocadas en la mejora de la productividad y que dan por supuesta la continuidad de las políticas públicas, sus objetivos y sus marcos institucionales de trabajo.

Lo más importante de la reforma presupuestaria no es conseguir una integración automática de la gestión por resultados 
y la evaluación en el proceso presupuestario, sino el tipo de resultados al que se presta atención por los sistemas de seguimiento y evaluación. El control y la evaluación de la gestión del gasto no debería sólo plantearse cuestiones del tipo single loop: ¿qué tal lo estamos haciendo?, ¿en qué medida estamos trabajando bien?, etc., sino además plantearse también las de tipo: double loop: ¿tiene sentido que hagamos esta tarea? (ARGY. RIS según referencia en F. LEEUw, 1996). La integración de la evaluación en el proceso presupuestario puede proporcionar grandes resultados en términos de reducción del déficit en la medida en que incorpore valoraciones relacionadas con el segundo orden de control o aprendizaje al que se refieren las cuestiones del tipo double loop.

La evaluación de programas y la capacidad de control en la Administración pública han evolucionado y pueden orientarse a responder las preguntas del tipo double loop y, por lo tanto, puede ser de utilidad para mejorar el proceso presupuestario. Pero el problema sigue siendo cómo acercar la acción evaluadora y los evaluadores a la acción de presupuestar y los decisores responsables de la asignación de recursos, y viceversa. Aunque las dificultades son grandes, las lecciones aprendidas hasta ahora en experiencias de reformas pasadas y las investigaciones realizadas ofrecen algunas ideas importantes para reiniciar los esfuerzos de integración de las funciones mencionadas (presupuesto, control y evaluación) con más posibilidades de avanzar hacia la coherencia y madurez del sistema presupuestario. A continuación se exponen algunas condiciones e iniciativas para llevar a cabo su acercamiento.

\section{Condiciones para mejorar la coherencia interna del sistema presupuestario}

La diferenciación entre el presupuesto, el control y la evaluación es necesaria, responde a criterios de especialización organizativa. El presupuesto, el control y la evaluación son en general actividades separadas, realizadas por personal de diferente preparación y con distintos intereses, valores y criterios de éxito profesional. El problema es que, como hemos visto en apartados anteriores, esta diferenciación no va normalmente acompañada de un esfuerzo de coordinación capaz de garantizar la coherencia del funcionamiento global del sistema presupuestario.

El diseño de una reforma orientada a la integración de estas funciones es complejo y depende de las características particulares de cada Administración. Pero, aunque en cada Admi- nistración el diagnóstico concreto sea distinto, siempre es útil conocer las líneas generales y condiciones de reforma que se desprenden de experiencias pasadas. A continuación se presentan una serie de medidas que pueden ayudar a mejorar la integración del sistema presupuestario. Estas medidas no pretenden ser una lista cerrada e infalible. Se trata más bien de una serie de condiciones, necesarias pero no suficientes, que hay que cumplir y obstáculos a superar según cada situación. Existe una interacción importante entre algunas de las propuestas que deberán tenerse en cuenta al diseñar el marco y estrategia de reforma.

\section{a) Condiciones técnicas}

División de responsabilidades clara y realista. Como primer paso es necesario rediseñar la estructura, procesos y funciones de los centros de control, evaluación y presupuesto, estableciendo una división clara de responsabilidades. Este reparto de competencias y roles puede ser diferente según la propia tradición de cada Administración, no hay un modelo ideal de aplicación universal. Lo importante es que el diseño elegido sea consensuado y acorde con la capacidad operativa y recursos asignados a cada centro participante.

Desarrollo de los sistemas de comunicación e interacción eficaz. La integración de estas funciones exige también disponer de sistemas de información y canales de comunicación entre los centros responsables que garanticen una interacción eficaz. Para ello, los instrumentos de recogida de información, los sistemas de mantenimiento y explotación de las bases de datos comunes o compartidas y los canales de intercambio de información necesita disfrutar de unos niveles aceptables de relevancia, fiabilidad y oportunidad en el momento de la toma de decisiones para la reasignación de recursos.

Disponibilidad de una masa critica de expertos en cada función. Un tercer requisito es la formación de evaluadores y expertos en control y presupuesto, distribuida entre los centros gestores y centros de presupuesto de manera coherente con el reparto de competencias elegido. Una preparación adecuada para la integración de estas funciones exige no sólo disponer de los conocimientos propios de cada especialidad, sino además las habilidades de comunicación y capacidad de entendimiento mutuo con los expertos de las otras funciones (aceptar interdependencias, identificar valores comunes, etc.).

Introducción de incentivos para el uso de los resultados del control y la evaluación. Es evidente que para que la integración tenga éxito el benéfico potencial que ofrece el control y la evaluación debería ser al menos compartido por los centros gestores y presupuestos. Igualmente habría que evitar o disminuir los efectos negativos de la normativa presupuestaria en el estilo de decisión o valores aplicados en la gestión presupuestaria. 
Por ejemplo, se podría evitar la devolución automática de todos los ahorros conseguidos con la evaluación, ya sea por recortes identificados o aumentos de productividad y eficacia, etc., concediendo una mayor discrecionalidad al gestor para su uso. Algunos países ya vienen practicando el uso de este tipo de incentivos desde los años noventa, principalmente los nórdicos (P. OVRELID, 1997).

\section{b) Condiciones político-institucionales}

Adaptar el marco institucional y las reglas de negociación presupuestaria. La modificación del estilo de decisión en la asignación de recursos de manera que tienda a tener en cuenta el valor añadido por los sistemas de control y evaluación exige además el cumplimiento de condiciones de naturaleza política. Es necesario adaptar el marco institucional y las reglas de negociación presupuestaria, tanto en el Gobierno como en el Parlamento. Aunque de manera implícita, los pactos de estabilidad presupuestaria son un ejemplo de adaptación del marco y las reglas del juego presupuestario. La sostenibilidad de su efecto va a depender de que se realicen esfuerzos de evaluaciones sistemáticas de las políticas públicas que consoliden los escenarios de disciplina de gasto agregado (Banco Mundial, 1997). Además de incentivar la disciplina presupuestaria, el marco político-institucional debe también fomentar una nueva cultura y estilo de gestión o tratamiento de conflictos presupuestarios (V. HAGEN, 1994; ZaPICO, 1995).

Adoptar un marco legal o establecer acuerdos institucionales para desarrollar la capacidad de evaluación. La formulación de acuerdos institucionales, con mayoría parlamentaria sostenible, o la adopción de la legislación apropiada para crear un marco formal e imagen política más favorable o propensa al uso de la evaluación de políticas públicas es también fundamental. Aunque esto no puede percibirse como una panacea, el inicio de un cambio de actitudes y estilos de gestión a favor de una mayor utilización y demanda de la evaluación de programas en el proceso presupuestario depende también de este tipo de medidas. Alguna experiencia en este sentido se ha realizado en varios países, por ejemplo, en Suecia y Francia, y además se ha demostrado que la evaluación tiende a estar más integrada en la gestión presupuestaria cuando ésta se realiza por órganos supremos e independientes de auditoria como ocurre en el Reino Unido (Gray, Jenkins y Segsworth, 1993; Derlien, 1990).

\section{Conclusiones}

Se ha analizado la importancia y posibilidades de integrar el control y la evaluación en el proceso presupuestario. Sin embargo, se ha observado cómo hasta ahora las experiencias conocidas en las que se ha intentado dicha integración su efecto no ha sido positivo y ésta ha resultado ser más difícil de conseguir de lo que se esperaba.

No obstante, a pesar de los débiles resultados, hay evidencias claras de que la evaluación se sigue percibiendo como de gran utilidad para el presupuesto y la gestión del gasto. Ésta puede facilitar el tránsito de una cultura de gasto, donde gobernar o gestionar bien se confunde con aumentar los créditos presupuestarios, a una cultura de resultados, donde el directivo público se centra y es responsable por el grado de eficiencia y eficacia alcanzado al gestionar los programas presupuestarios La integración o coherencia del proceso presupuestario puede proporcionar importantes beneficios en términos de reducción relevante y sostenible de costes si incorpora valoraciones y proporciona información relacionadas con el segundo orden de control o evaluación (double loop learning), evitando que gran parte de los recursos públicos se orienten a resolver problemas que han dejado de existir o se presten servicios que la sociedad ya no valora o necesita.

Pero la integración del sistema presupuestario no puede entenderse como un mecanismo para imponer unos criterios técnicos supuestamente objetivos y neutros en la asignación de recursos. Los resultados del control y la evaluación pueden aspirar a ser un factor importante pero no el único a tener en cuenta en el proceso presupuestario. La utilidad de los sistemas de control y evaluación se verá potenciada en el proceso presupuestario en la medida en que el Ministerio de Finanzas y/o el Gobierno desarrollen su capacidad de confrontar y gestionar con eficacia los conflictos que surgen al identificar necesidades de reasignar recursos a nuevas prioridades. La evaluación de programas y las técnicas de decisión presupuestaria deben intentar ir más allá del tradicional criterio marginalista de maximizar la eficiencia de los recursos disponibles. La integración del control y la evaluación en el proceso presupuestario puede proporcionar grandes resultados, incluso en términos de reducción del déficit en la medida en que incorpore como criterios de éxito la adaptación y el 'aprendizaje' en el diseño y desarrollo de las políticas públicas.

El control y la evaluación de progtamas no pueden entenderse ni deben aplicarse de manera mecánica en el proceso presupuestario. La expectativa de reasignar recursos automáticamente de acuerdo a los resultados de los sistemas de seguimiento y evaluación de las políticas públicas es ingenua. No obstante, si se cumplieran las condiciones identificadas en este trabajo, hay ciertas posibilidades de que puedan ser utilizados como un factor más entre otros de los que influyan en el proceso presupuestario. Además, el control de gestión y la evaluación de programas es útil para que los centros gestores adapten su comportamiento presupuestario a una situación en la que se 
necesita reasignar recursos dentro de un límite o tope de gasto impuesto desde el Ministerio de Finanzas.

Pero el verdadero valor añadido de los sistemas de seguimiento y evaluación de programas reside en el propio proceso de valoración más que en sus resultados, entendido como un proceso interactivo y abierto al aprendizaje por parte del controlador o evaluador y del gestor. Gran parte del beneficio de una buena evaluación debe haberse materializado antes de formularse el informe final. El proceso de evaluación podría y debería orientarse a la consolidación de los canales de comunicación e interacción entre decisores, participantes y grupos de interés (stake-bolders) en general del programa presupuestario en cuestión. Esto aumentaría la coherencia interna del presupuesto ya que permitiría reducir la incertidumbre «innecesaria» o evitable, que se plantea en todo proceso de asignación de recursos cuando no se gestionan de manera apropiada las interdependencias y conflictos propios de este proceso (WIL DAVSKY y ZAPICO, 1993).

La coherencia o integración del proceso presupuestario es compleja y no hay soluciones preestablecidas. Es necesario realizar el diagnóstico de cada Administración para proponer mejoras concretas. Pero, las experiencias vividas por diversos países nos han permitido al menos identificar las principales condiciones generales, tanto de tipo técnico como político, que pueden plantearse como requisitos necesarios para progresar en el mencionado esfuerzo de integración del sistema presupuestario.

* Subdirector General de Análisis y Evaluación de las Políticas de Gasto. Dirección General de Presupuestos. Ministerio de Hacienda.

Bibliografía

BLONDAL, J. (2000), «Institutional Arrangements and the Control of Public Expenditure in OECD Member countries», en ASIP XXVII International Seminar on Public Budget, Madrid.

BotNer, S. (1991), «Trends and Developments in Budgeting and Financial Management in Medium-sized Cities of the United States», en Public Budgeting and Financial Management, vol. 3 (2), Marcel Dekker: 443.

Cothran, D. (1993), «Entrepeneurial Budgeting: An Emerging Reform?», en Public Administration Reform, septiembre-octubre.

CARTER, Neil, (1989), «Performance Indicators: "back seat driving" or "hands off" control?», en Policy and Politics.

FlynN, N., y StreHL, F. (1996), Public Sector Management in Europe, London: Prentice Hall.

GibSON, J., y BUTLEY, R. (eds.) (1992), «Financing European Local Governments», en Local Government Studies, vol. 18, núm. 4, winter, London.

US General Accounting Office (1993), «Performance Budgeting», AFMD93-41, Washington DC.

Gradin, A. (1996), «Introduction», en Acbieving Better Value from European Community Funds, Bruselas: FEE.

Gray, A.; Jenkins, B., y Segsworth, B. (1993), Budgeting, Auditing and Evaluation, Transaction, P. New Brunswick (USA).

Hofstede, G. (1981), «A Management Control of Public and Not-For-Profit Activities», Accounting Organizations and Society, 6(3).

Kane, J. (2000), «El Presupuesto como Instrumento de Política Publica», en Análisis Local, núm. 2.

Landau, M., y R. Stout, Jr. (1979), «ATo Manage is Not to Control: Or the Folly of Type II Errors», Public Administration Review, marzo-abril.
LIIKANEN, E. (1996), «Improving Financial Management in the EU», en Acbieving better value from European Community Funds, Belgium: FEE.

Lonco, Francisco (1998), «Política y Gerencia Pública en los Gobiernos Locales», en X. Sisternas, Estado de la Gerencia Publica en la Administración Local, Bar. celona: Fundación Carles Pi i Sunyer.

Mayne, J., y ZaPico, E. (1997), Monitoring Performance in the Public Sector, Transaction P. New Brunswick (USA).

Metcalfe, L., y Ruchards, S. (1990), Improving Public Management. 2. a ed., Sage.

MetCalfe, L. (1993), «A Public Management: From Imitation to Implementation», en J. Koolman (ed.), Modern Govermment, Sage.

Ministerio de Hacienda (2000), Proyecto de Ley de Estabilidad Presupuestaria, Documento interno.

Ministerio de Administraciones Públicas (2000), Libro Blanco para la Mejora de los Servicios Publicos, febrero, Madrid: MAP.

Novick, D. (1969), «The Origen and History of Programe Budgeting», en Pro. grame Budgeting, Halt, Rinehart.

OECD (1991), «Serving the Economy better», PUMA (91) 10, working draft núm. 3.

— (1994), «Performance measurement in Government», Paris, núm. 3.

- (1994), «Performance measurement in Government», Paris, núm. 5.

- (1995), «Budgeting for Results: Perspectives on Public Expenditure Mana. gement», Paris. 
- (1996), Modem Budgeting. PUMA (96).

- (1997), «In Search of Results: Performance Management Practices», Paris.

- (1997), «Managing Across levels of Government», Paris.

- (1999), «Performance Contracting», PUMA (99) 2, Paris.

OlsEn, J., y PETERS, G. (1996), Lessons from Experience: Experiential leaming in Administrative Reform in Eight countries, Oslo: Scandinavian University Press.

OrTigueira, Manuel (1989), «El escenario internacional de la presupuestación pública: especial consideración de la imagen actual de la Institución Presupuestaria Local», en Temas de Administración Local, núm. 37, Granada: Centro de Estudios Municipales.

OvreLiD, P. (1997), «The impact of linking evaluation with Budgeting, Directorate of Public management in Norwayn, paper presented at the Conference on Evaluation in Oslo, EES.

SchICK, A. (1978), «The Road to PPB», en A. Hyde y J. SHAFrTt, Government Budgeting, Moore PC.

ScHICK, Allen (1986), «Macro budgetary adaptations to fiscal stress in industrialized democracies», en Public Administration Review, marzo-abril.

- (1990), «Budget for Results: recent development in five industrialized countries», en Public Administration Review, enero-febrero.

Stockman (1984), El triunfo de la Politica sobre el Presupuesto, OMB, President of the US.
Syzve Trosa (1997), «Capítulo 5...», en J. Mayne y E. Zapico, (eds.), Monitoring Performance in the Public Sector: Future Directions from Intermational Experience, New Jersey: Transaction.

Verheijen, T., y CoOmbes, D. (eds.) (1998), Innovations in Public Management, Cheltenham, RU: Edward Elgar.

Wudavsky, A. (1975), Budgeting: A Comparative Theory of Budgetary Processes, Little Brown \& Co.

- (1988), «A Cultural Theory of Budgeting», International Joumal of Public Administration, 11 (6).

Wudavsky, A., y ZaPICO, E. (eds.) (1993), National Budgeting for Economic and Monetary Union, London: Martinus Nijhoff P.

ZAPICO, E. (1995), «Proceso presupuestario como un proceso de negociaciones complejas», en Cuadernos de Relaciones Laborales, Madrid: UCM.

- (1995), Administración Financiera y la Nueva Gerencia Pública, Madrid: IEF.

- (1998), «La Integración de las Políticas Publicas en el Proceso Presupuestario", en la Revista GAPP, enero-agosto, núms. 11 y 12.

- (2001), «Hacia un nuevo Modelo de Reforma Presupuestaria para el Control y Gestión Estratégica del Gasto en Contexto de Complejidad e Incertidumbre», presentado en la Conferencia sobre Control de Gestión en las AAPP, organizada por el Institute for International Research en Madrid.

Zapico, E., y MaYne, J. (1995), «Nuevas Perspectivas para el Control de Gestión y la Medición de Resultados», en la Revista GAPP, mayo-agosto, núm. 3. 